\title{
Non-equilibrium Wall Deposition of Inertial Particles in Turbulent Flow
}

\author{
John R. Schmidt • Jost O. L. Wendt • Alan R. Kerstein
}

Received: 2 April 2009 / Accepted: 9 October 2009 / Published online: 27 October 2009

(C) The Author(s) 2009. This article is published with open access at Springerlink.com

\begin{abstract}
Non-equilibrium effects resulting from the slow relaxation of inertial particles to statistical equilibrium with flow fluctuations in turbulence are known to have important consequences, but they are not readily incorporated into models. Here, a simple analysis of these effects predicts $-2 / 3$ power-law dependence of the particle deposition rate on Stokes number (normalized particle inertia) in the far field of a confined turbulent flow, and a weaker near-field dependence. Near-field measurements and numerical simulations exhibit this weaker dependence, as do models that are generally viewed as validated by this result, but the models fail to capture the newly identified far-field behavior due to their equilibrium assumptions. Quantification of these qualitative observations is obtained by incorporating particle response to fluid motion into 'one-dimensional turbulence' (ODT), a stochastic computational model of turbulence.
\end{abstract}

Keywords Multiphase flow · Deposition · Turbulence $\cdot$ Stochastic model

\footnotetext{
J.R. Schmidt · J.O.L. Wendt

Department of Chemical and Environmental Engineering, University of Arizona, Tucson, AZ 85721, USA

Present address:

J.R. Schmidt

Army Research Laboratory, Aberdeen Proving Ground, MD 21005, USA

e-mail: jschmidt@arl.army.mil

Present address:

J.O.L. Wendt

Department of Chemical Engineering, University of Utah, Salt Lake City, UT 84112, USA

e-mail: jost.wendt@utah.edu

A.R. Kerstein ( $₫)$

Combustion Research Facility, Sandia National Laboratories, Livermore, CA 94551-0969, USA

e-mail: arkerst@sandia.gov
} 


\section{Introduction}

Modeling of particle-laden turbulent flow confronts the difficulty that the response of inertial particles to fluid motion is time lagged, so the kinematic state of the particle field is sensitive to past history as well as the current flow state. The coupled particle-fluid system can nevertheless be in a state of statistical quasi-equilibrium, which is here termed equilibrium in accordance with the terminology in the multiphase-flow literature. However, equilibrium in this restricted sense does not hold in many cases. The recognized importance of nonequilibrium effects has motivated heuristic modifications of equilibrium-based models, but existing modeling frameworks do not readily accommodate a fundamentally sound representation of non-equilibrium particle-fluid states [24, 25].

Equilibrium-based models have significant capabilities, including the reported prediction of wall deposition rates of high-inertia as well as low-inertia particles [7, 24, 28]. Nonequilibrium effects are strongest at high inertia (i.e., slow particle response to fluid motion), so the reported agreement with measurements and numerical simulations in this regime is especially noteworthy. However, it is shown here that this apparent agreement is the result of a fortuitous cancellation of errors.

The first error is the previously unnoticed inability of equilibrium-based models to capture a leading-order effect of non-equilibrium in the high-inertia regime. This effect is identified here by means of a simple scaling analysis of this regime. The second error is the interpretation of transient evolution in numerical simulations as long-time asymptotic behavior. This point is addressed here using a stochastic computational model of turbulence, denoted 'one-dimensional turbulence' (ODT) [12], which is extended for this purpose to incorporate particle-flow interactions. It would be difficult if not impossible to obtain experimental confirmation of these results and inferences, but a definitive three-dimensional (3D) numerical simulation, guided by the present results, should be attainable.

Section 2 describes the specific flow configuration that is studied and its relevant features. The high-inertia regime is analyzed in Sect. 3. ODT and its extension to particle-laden flow are described in Appendices A and B, respectively, and computed results are presented and interpreted in Sect. 4.

\section{Phenomenology of Deposition from Particle-laden Turbulence}

To tie the present study to previous work, the specific focus is particle deposition on the side walls of fully developed turbulent channel flow, which is statistically homogeneous in the streamwise (mean-flow) direction and statistically stationary. Several simplifying assumptions are adopted that are consistent with previous modeling and numerical simulation studies but differ in several respects from experimental conditions. Although comparisons to experimental results are shown for completeness, the lack of direct comparability to analysis, models, and simulations (present and previous) limits their role relative to comparisons among models and simulations. Nevertheless, it is shown in Sects. 3.3, 4, and 5 that novel insights are gained with regard to the interpretation of numerical simulation results, the realm of applicability of various models, and the comparison of both to experimental data.

Particles are assumed to be dynamically passive, i.e., they do not affect the flow or each other. Their response to the flow is idealized as Stokes drag in the scaling analysis, supplemented by an empirical particle-Reynolds-number correction in some of the computations (see Appendix B). Gravitational effects are omitted. A particle that hits a wall is deemed to be deposited there and is no longer present. (The experiments use droplets that form a 
film when they hit the wall, and are performed in pipes rather than channels.) The accuracy of these idealizations decreases with increasing particle inertia, but they are useful for elucidating the underlying physics provided that the results are interpreted with due caution.

The following fluid-mechanical terminology is used. The fluid (gas) flow is parameterized by the channel half-height $h$, the kinematic viscosity $v$, and either the bulk flow velocity $u_{b}$ or the friction velocity $u_{\tau}=\sqrt{v|d \bar{u} / d y|_{h}}$, where $\bar{u}(y)$ is the mean streamwise $(x)$ velocity expressed as a function of the wall-normal coordinate $y$ and $h$ denotes evaluation at a wall $(y= \pm h) . u_{\tau}$ is more fundamental than $u_{b}$ in that $u_{\tau}$ can be expressed in terms of $v$, the (constant) fluid density $\rho_{f}$, and the imposed mean streamwise pressure gradient $d p / d x$ through momentum conservation, but $u_{b}$ for given flow setup is an empirical quantity, not yet derived from first principles.

The only relevant particle property is the aerodynamic time constant $\tau_{p}$; see (19). Although finite particle radii are used in the ODT simulations for consistency with comparison cases, they are so small that the results are indistinguishable from results for nominal point particles. $\tau_{p}$ and fluid parameters are combined to form the particle Stokes number (wallnormalized time constant) $\tau_{p}^{+}=\tau_{p} u_{\tau}^{2} / \nu$. (In general, superscript + denotes wall normalization.)

The local (in time) particle deposition rate is expressed as a deposition velocity $V_{d}$, defined as depositions per unit wall area per unit time divided by the instantaneous volumeaveraged particle concentration, here allowing for time variation of that concentration. (Time rather than $x$ is the independent variable because temporal evolution of the streamwisehomogeneous flow is considered; see Sect. 4.4 and Appendix A.) The corresponding dimensionless quantity is $V_{d}^{+}=V_{d} / u_{\tau}$.

Measurements of the dependence of $V_{d}^{+}$on $\tau_{p}^{+}$exhibit three distinct regimes termed the diffusional-deposition, diffusional-impaction, and inertia-moderated regimes, with transitions at $\tau_{p}^{+}=0.3$ and 30 respectively. In the inertia-moderated regime, measurements up to $\tau_{p}^{+}$of order $10^{3}$ suggest a barely perceptible (relative to experimental uncertainty) decline of $V_{d}^{+}$with increasing $\tau_{p}^{+}[15,28]$. The behavior in this $\tau_{p}^{+}$range (and at smaller $\tau_{p}^{+}$) is reproduced by closure modeling of the ensemble-averaged governing equations [7, 25, 28]. It is recognized that the decline at large $\tau_{p}^{+}$is due to the decreasing effectiveness of turbulence in inducing lateral deflections of particles as particle inertia increases.

Large-eddy simulation (LES) of pipe-flow deposition extending to higher $\tau_{p}^{+}$values (up to $10^{4}$ ) [26] suggests an acceleration of the declining trend for $\tau_{p}^{+}>10^{3}$. There does not appear to be subsequent discussion of this observation in the literature, leaving open the question of whether the aforementioned models capture this effect.

A physical basis for sharper decline is identified here using a straightforward scaling analysis that accounts for non-equilibrium effects. A computational model is then used to quantify the predicted behavior and relate it to the results of previous studies.

Both of these approaches indicate that the onset of the asymptotic behavior occurs only after a long transient relaxation period. Previously reported weak $\tau_{p}^{+}$dependence seen at large $\tau_{p}^{+}[1,27,28]$ might be partially the result of observation during the initial transient (corresponding to the near field in spatially developing flow) rather than the ultimate statistically steady evolution. This suggests that further computational (and possibly experimental) study might be needed in order to distinguish asymptotic behavior from initial transients. Previous analysis by Graham [5] has analogous implications, albeit without addressing the particular regimes and mechanisms considered here. 


\section{Analysis of High-inertia Deposition}

\subsection{Scaling Analysis}

Turbulent channel flow is characterized by three velocity scales: the bulk velocity $u_{b}$, a turbulent fluctuation amplitude ('large-eddy velocity') $u^{\prime}$, and the friction velocity $u_{\tau}$. The relationship between $u^{\prime}$ and $u_{\tau}$ in channel flow is discussed in Sect. 3.2.

The other governing quantities are $h, \tau_{p}$, and $v . h / v$ times each of the respective velocity scales defines bulk, turbulent, and friction Reynolds numbers $R e_{b}, R e^{\prime}$, and $R e_{\tau}$. High turbulence intensity (based on any of these Reynolds numbers) is assumed. Stokes drag is assumed with no particle-Reynolds-number correction.

To distill the essential physics governing the parameter dependences of $V_{d}^{+}$in the limit of high particle inertia, the lateral (wall-normal) structure of the channel flow, e.g. the $y$ dependence of $u^{\prime}$, is neglected, so in effect, the flow is idealized as homogeneous, isotropic, statistically steady turbulence with mean streamwise flow velocity $u_{b}$.

Various particle initial conditions are considered. It is convenient to begin by assuming that particles traveling at given streamwise $(x)$ velocity $U_{0}$ are introduced, with zero lateral $(y)$ or spanwise $(z)$ velocity, at the nominal flow mid-plane, $y=0$. For now, $U_{0}$ is assumed to be much larger than flow velocity scales, but later this assumption is relaxed. Deposition is deemed to occur upon first passage through one of the planes $y= \pm h$.

For high particle inertia, it is shown in Sect. 3.3 that the particle deposition time $t_{d}$ is much less than $\tau_{p}$, and consequently that the particle streamwise velocity $U$ remains large and its lateral velocity $V$ remains small compared to characteristic flow velocities. Therefore the streamwise particle slip $S_{U}=U-u$ is of order $U_{0}$ and the lateral slip $S_{V}=V-v$ is of order $u^{\prime}$. Here, $(u, v, w)$ and $(U, V, W)$ denote the fluid and particle velocity vectors, respectively, in terms of the streamwise, lateral, and spanwise components. Where the arguments (location and time) of velocity components are not obvious from the context, they are shown explicitly. Spanwise slip is immaterial under the stated assumptions and therefore is not considered.

Though the idealized homogeneous flow is unconfined, the effect of lateral confinement is implicitly introduced by taking the turbulence integral scale (largest eddy size) to be of order $h$, so the large-eddy time scale is $T \sim h / u^{\prime}$. The particle eddy-crossing time $t_{c}$ is of order $h / U_{0}$. Based on Stokes drag (see Appendix B), the particle velocity change in a given direction while crossing an eddy scales as $S t_{c} / \tau_{p}$, where $S$ is the corresponding slip component, $S_{U}$ or $S_{V}$. The particle lateral velocity change caused by a large-eddy traversal is then $\Delta V \sim u^{\prime} t_{c} / \tau_{p}$.

During a time interval $t \gg t_{c}$, a particle is subject to order $t / t_{c}$ independent velocity increments due to these eddy effects. (This is based on $U_{0} \gg u^{\prime}$. If this does not hold, then $T$ is the relevant time scale, replacing $t_{c}$ and thus eliminating dependence on $U_{0}$.) The regime of interest is the limit of large $\tau_{p}$ in which the individual velocity increments $\Delta V$ are small. Under these conditions, the time evolution of the velocity scale $V$ is a diffusion process that obeys the scaling $V(t) \sim \Delta V\left(t / t_{c}\right)^{1 / 2} \sim\left(u^{\prime} / \tau_{p}\right)\left(t_{c} t\right)^{1 / 2}$, and hence $d V^{2} / d t \sim D$, where the velocity diffusion coefficient $D$ obeys $D \sim u^{\prime 2} t_{c} / \tau_{p}^{2}$.

This diffusion picture omits a damping mechanism that causes $V(t)$ to transition eventually to a stationary random process. In Sect. 3.3, this transition is shown to occur at a time far beyond the deposition time $t_{d}$, demonstrating both that the diffusion picture is valid for this regime and that high-inertia deposition is a non-equilibrium process.

The deposition time is estimated to be the time $t$ at which $h=t V(t)$, giving

$$
t_{d} \sim\left(h^{2} \tau_{p}^{2} / u^{\prime 2} t_{c}\right)^{1 / 3} .
$$


The definition of the deposition velocity $V_{d}$ in Sect. 2 implies that $V_{d}$ scales as $h / t_{d}$, giving

$$
V_{d} \sim\left(h u^{\prime 2} / t_{c}\right)^{1 / 3}\left(t_{c} / \tau_{p}\right)^{2 / 3} .
$$

This result exhibits $-\frac{2}{3}$ power-law dependence of the deposition rate on the particle time constant, the key physical result, which is now interpreted in the context of channel flow.

\subsection{Implications for Channel Flow}

Two salient features of channel flow that are omitted from the foregoing analysis are the presence of mean shear that drives turbulent motions and the inhibition of turbulent transport near the walls. One manifestation of the complexity of the resulting flow is experimental [2] and computational [8] evidence that the maximum (with respect to $y$ ) of $u^{\prime}$ in turbulent channel flow scales as $\left(u_{b} u_{\tau}\right)^{1 / 2}$. It is difficult to formulate a statistical description of nearwall flow that is consistent with this, let alone analyze particle response to this flow, so no attempt is made here to generalize the model of Sect. 3.1 to incorporate lateral flow inhomogeneity. Therefore the model has the same degree of applicability to pipe flow as to channel flow.

Treating $u^{\prime}$ as a single velocity scale rather than as a function of $y$ is in any case an idealization. For simplicity, $u^{\prime} \sim u_{b}$ is assumed, but the consequence of instead assuming $u^{\prime} \sim u_{\tau}$ is noted below. For either case, $V_{d}$ is normalized by the friction velocity $u_{\tau}$, both because it is the customary normalization at lower inertia, for which the near-wall flow controls the deposition rate, and because this normalization is found to collapse the high-inertia numerical results of Sect. 4, although the reason for this is unclear due to the aforementioned complications.

Division of (2) by $u_{\tau}$ and substitution of $h / U_{0}$ for $t_{c}$ and $u_{b}$ for $u^{\prime}$ give

$$
V_{d}^{+} \sim\left(U_{0}^{+}\right)^{-1 / 3} \operatorname{Re}_{b}^{2 / 3}\left(\tau_{p}^{+}\right)^{-2 / 3},
$$

which identifies the $\tau_{p}^{+}$dependence of $V_{d}^{+}$. Dependence on $u_{b}$ is eliminated using the Blasius resistance formula, long regarded as empirical but recently derived from a more fundamental perspective [4]. The Blasius formula, $f \sim R e_{b}^{-1 / 4}$, where the friction factor $f$ scales as $\left(R e_{\tau} / R e_{b}\right)^{2}$, implies $R e_{b} \sim R e_{\tau}^{8 / 7}$ and thus

$$
V_{d}^{+} \sim\left(U_{0}^{+}\right)^{-1 / 3} \operatorname{Re}_{\tau}^{16 / 21}\left(\tau_{p}^{+}\right)^{-2 / 3} .
$$

The exponent $\frac{16}{21}$ is replaced by $\frac{2}{3}$ if $u^{\prime} \sim u_{\tau}$ is assumed rather than $u^{\prime} \sim u_{b}$. Thus, the exponent varies only slightly over a range of possible behaviors that bracket the observed scaling.

For the cases considered in Sect. 4, $U_{0}$ is not large, so $t_{c}$ is replaced by $T$ in (2). This eliminates dependence on $U_{0}$, giving

$$
V_{d}^{+} \sim \operatorname{Re}_{\tau}^{p}\left(\tau_{p}^{+}\right)^{-2 / 3}
$$

where $p=\frac{2}{3}$ for $u^{\prime} \sim u_{\tau}$ and $p=\frac{5}{7}$ for $u^{\prime} \sim u_{b}$, indicating $p \approx 0.7$ within the plausible range of behaviors.

The high-inertia results of Sect. 4 based on a stochastic computational model of channelflow deposition indicate little if any dependence of $V_{d}^{+}$on $R e_{\tau}$, suggesting that neglected flow structure counteracts the $R e_{\tau}$ dependence implied by the analysis. Yet those results agree with the predicted dependence on $\tau_{p}^{+}$, indicating that the analysis leading to (5) captures fundamental features of high-inertia deposition. Henceforth, discussion of (5) refers collectively to its variants based on assumptions about $U_{0}$ and $u^{\prime}$. 


\subsection{Initial Conditions, Transients, and Equilibration}

Based on (1), $t_{d} / \tau_{p} \sim\left(\frac{T^{2}}{t_{c} \tau_{p}}\right)^{1 / 3}$, which is much less than unity in the limit of large $\tau_{p}$. The time required for $V(t)$ to transition from transient growth to equilibration is much longer than $t_{d}$. This transition occurs when $V^{2}$ reaches its equilibrium value determined by $d V^{2} / d t \sim D-V^{2} / \tau_{p}=0$. Here, the sink term $V^{2} / \tau_{p}$ omitted from the derivation of (2) is included. This is an idealized (but adequate for present purposes) representation of the decay of particle velocity fluctuations, governed by the particle response time scale, in the absence of forcing of the fluctuations. The familiar [24] equilibrium result

$$
V^{2} \sim D \tau_{p} \sim u^{\prime 2} t_{c} / \tau_{p}
$$

is thus obtained. Until the onset of equilibration, $V^{2}(t) \sim D t$, showing that the elapsed time until equilibration is $\tau_{p}$, which is much larger than the estimated deposition time $t_{d}$ in the limit of interest. Based on (2), $V\left(t_{d}\right)$ is smaller than the equilibrium value by the factor $\left(\frac{T^{2}}{t_{c} \tau_{p}}\right)^{1 / 6}$. These estimates indicate that the transient analysis is self-consistent, hence confirming the non-equilibrium nature of high-inertia deposition under the stated assumptions.

The dependence of the scaling of $V_{d}^{+}$on $V(0)$ is considered. Suppose that $V(0)$ is nonzero. Owing to the smallness of the deviations from ballistic particle trajectories for large $\tau_{p} / t_{c}$, the deposition time $t_{d}$ for $V(0) \neq 0$ is $h / V(0)$ unless $V(0)$ is small enough so that $h / V(0)$ is larger than $t_{d}$ given by (1). Thus, (1) is an upper bound on the deposition time, which can be much smaller for nonzero $V(0)$.

More generally, assume a distribution of particle $V(0)$ values. $h / V(0)$ will be smaller than $t_{d}$ for most particles, so most of the deposition will be ballistic in character, and thus deposition is governed primarily by initial conditions and is more rapid than predicted by (5). However, the (generally small) fraction of particles for which $V(0)<h / t_{d}$ deposit by the non-equilibrium mechanism analyzed here, and accordingly their deposition rate is governed by (5). This indicates that high-inertia deposition for the conditions considered (Stokes drag, zero gravity), is primarily a transient, case-specific process, and that phenomenology of a more general nature arises only as a far-field (downstream) asymptote. Implications with regard to the interpretation of measurements and simulation results are considered in Sect. 4.4.

It is assumed in the analysis that particles are introduced at the channel mid-plane, but the far-field analysis is unchanged if a more general initial spatial distribution is assumed. Only particles introduced at a distance from the wall that is much less than $h$ will deposit more rapidly than the analysis predicts. The near-field transient deposition due to nonzero $V(0)$ may be strongly affected by the initial spatial distribution. This dependence is likewise considered in Sect. 4.4.

The analysis highlights the non-equilibrium character of particle response to turbulent fluctuations in the regime of interest. Nevertheless, the equilibrium condition, (6), is adopted in closure-type modeling of particle deposition in turbulent channel flow [24, 28]. The deficiency of this assumption is recognized and a compensatory modification has been attempted, but the closure framework does not readily accommodate a fundamentally sound representation of non-equilibrium [24, 25]. Reflecting this, closure modeling predicts less rapid decrease of $V_{d}^{+}$with increasing $\tau_{p}^{+}$than is indicated by (5). The milder dependence is found to be consistent with experimental and numerical simulation results. However, these results reflect case-specific near-field transient phenomena rather than the far-field asymptote, as shown in Sect. 4.4. Therefore, the consistency of these results with closure-model predictions is not as clear a validation of model assumptions as is commonly supposed. 


\section{Computed Results and Interpretation}

\subsection{Modeling Approach}

The analysis in Sect. 3 implies that high-inertia deposition is challenging to investigate either experimentally or computationally. The slow relaxation of transients requires a large experimental apparatus in order to observe the far-field behavior, as emphasized by Graham [5]. Likewise, it requires large spatial domains in numerical simulations (or long run times, in the more usual temporally developing configuration).

One of the authors has developed a cost-effective method for simulating turbulence and its coupling to other processes using a modeling strategy based on reduction of spatial dimensionality. A 1D spatial representation is employed. It has been shown that a stochastic simulation model formulated in 1D provides a representation of turbulence phenomenology that is useful in many respects $[11,14]$. This approach, denoted 'one-dimensional turbulence' (ODT), is outlined in Appendix A and generalized in Appendix B for simulation of inertial particles in turbulence.

Here, numerical results of ODT simulations of channel-flow deposition are compared to the results of the scaling analysis, to the results of previous experiments and numerical simulations, and to the predictions of other models. These comparisons support the inferences drawn in Sect. 3 and provide further insights into the relationships among models, measurements, and simulations of high-inertia deposition.

\subsection{Parameter Assignment}

For this application, model parameters are assigned the values previously determined for single-phase turbulent channel flow (see Appendix A). Particle-eddy interaction introduces an additional parameter $\beta$, which is the coefficient in the relation $t_{E}=\beta \tau$ that relates the eddy lifetime $t_{E}$ to the eddy-turnover time $\tau$ that determines the eddy frequency (see Appendix B).

$\beta$ is evaluated by matching ODT results to the results of direct numerical simulations (DNS) in a $\tau_{p}^{+}$range that is below the high-inertia regime. After $\beta$ is fixed in this manner, all ODT results for the high-inertia regime, including transitional behavior (with respect to both $\tau_{p}^{+}$variation and transient relaxation) are predictions that are directly comparable to previous results.

On this basis, $\beta$ is adjusted to give the best agreement between ODT deposition simulations and DNS [17] of channel-flow deposition for $\tau_{p}^{+}$values ranging from 2 to 6 . As in the DNS, Stokes drag is used in the ODT simulation. Initial conditions and other details of this and other simulated cases are discussed in Appendix B. $R e_{\tau}=125$ in the DNS, but the ODT comparison case is $R e_{\tau}=180$ because the high- $R e$ phenomenology implicit in ODT model assumptions is marginally valid at $R e_{\tau}=180$ and problematic at lower $\operatorname{Re}$ [21]. $R e_{\tau}$ sensitivities presented in Sect. 4.3 indicate that the difference between the DNS and ODT $R e_{\tau}$ values should not noticeably impact the comparison.

The best agreement is obtained for $\beta=0.3$, which is used in all subsequent ODT simulations. For this value, the comparison to the DNS data is shown in Fig. 1. It should be noted that the DNS results are subject to considerable statistical uncertainty; in particular, the lowest data point is based on a single deposition event. 


\subsection{Stokes-number Dependence of Deposition}

Although the present focus is deposition at large $\tau_{p}^{+}$, it is useful to consider smaller $\tau_{p}^{+}$values as well, both for parameter evaluation, as in Sect. 4.2, and to provide a general context for interpretation of model results. Model results for the range of $\tau_{p}^{+}$values considered here are shown in Fig. 2 for the same conditions (Stokes drag, $\operatorname{Re}_{\tau}=180, \beta=0.3$ ) as in Fig. 1, along with selected comparison cases.

The drag law used to obtain the low-inertia DNS $\left(R e_{\tau}=125\right)$ results [1] shown in Fig. 2 includes the Cunningham slip factor, Brownian motion force, Saffman lift, and wall effects on both drag and lift, none of which are included in the present model formulation (although they can be incorporated and will be included in future work). Accordingly, the low-inertia data is not a suitable basis for setting the model parameter $\beta$ in the present formulation because this parameter would subsume the error caused by the use of Stokes drag. This further motivates the choice of the $\tau_{p}^{+}$range used to evaluate $\beta$ in Sect. 4.2.

The disparity between the low-inertia measurements [15] shown in Fig. 2 and the DNS is greater than the disparity between model results and either of these. The experiment, involving droplet deposition in tubes, was subject to complications, such as droplet shape

Fig. 1 Dependence of normalized particle deposition rate $V_{d}^{+}$on Stokes number $\tau_{p}^{+}$in turbulent channel flow, using Stokes drag. o, ODT for $\beta=0.3$, $R e_{\tau}=180 ; \bullet$, DNS [17] for $R e_{\tau}=125$

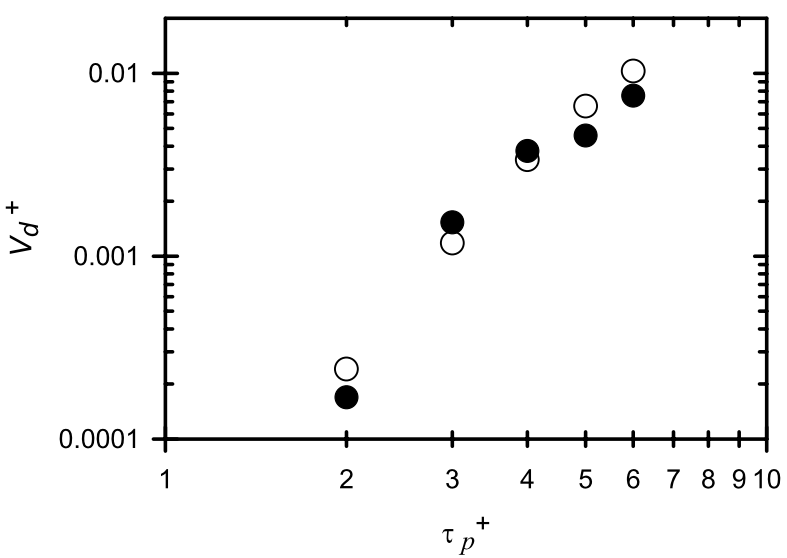

Fig. 2 Comparison of ODT deposition rates, for the same conditions as Fig. 1 runs, to simulations and measurements. ○, ODT; $\square$, DNS [1]; + and $\times$, measurements [15] for flow Reynolds numbers 10,000 and 50,000 , respectively;

•, measurements [3]. Line slopes: ,$--2 / 3 ;---,-1 / 2$

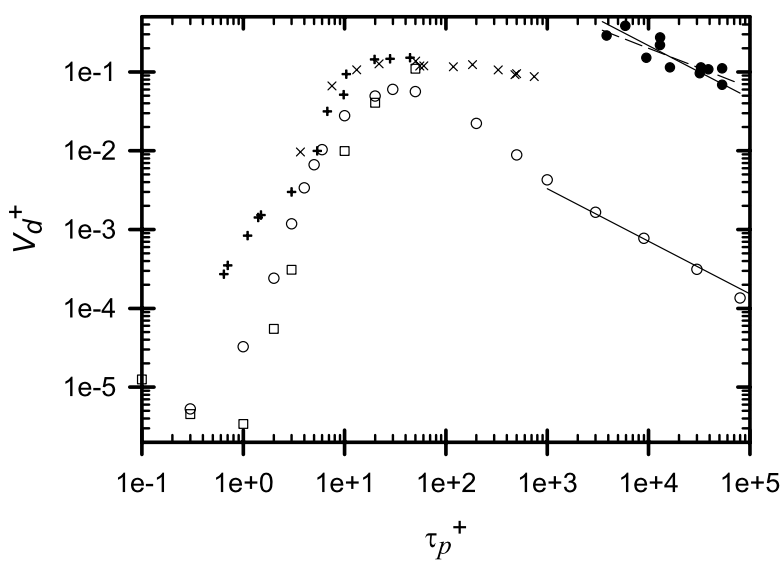


Fig. 3 ODT results of Fig. 2 in the format of that figure, and additional ODT results for $R e_{\tau}=640(\square)$ and $1200(\diamond)$. ,$--2 / 3$ slope. Otherwise, same symbol definitions as in Fig. 2

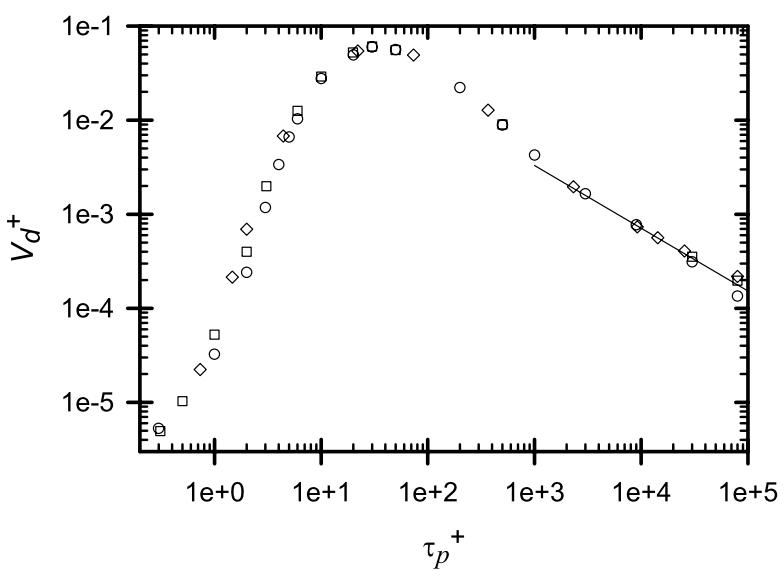

distortion and polydispersity, that can plausibly account for the differences between the DNS and experimental results [1].

Despite the complications, the overall increase of $V_{d}^{+}$with increasing inertia, followed by leveling (not seen in the DNS data, but an eventual necessity because the ballistic deposition rate is finite) is well understood [28]. The low-inertia trend reflects the role of inertia in enabling particles to cross fluid streamlines, as is required for deposition when effects of finite particle radius are negligible. The resulting increase of deposition with increasing inertia saturates when the deposition becomes nearly ballistic and hence relatively insensitive to further increase of inertia.

In Sect. 3, analysis of high-inertia deposition indicates that the ballistic mechanism is a near-field transient that is followed by far-field relaxation to a less effective mechanism, resulting in $\left(\tau_{p}^{+}\right)^{-2 / 3}$ dependence of $V_{d}^{+}$. The high-inertia results in Fig. 2 are consistent with the interpretation that the measurements by Liu and Agarwal [15] correspond to the near-field ballistic regime and model results correspond to the far-field regime. Additional evidence supporting this interpretation is examined in Sect. 4.4.

Also shown in Fig. 2 are measurements [3] that have been interpreted [16] as a decreasing trend following an empirical $-\frac{1}{2}$ power law. It is noted in passing that the predicted $-\frac{2}{3}$ high-inertia far-field scaling is an equally good statistical inference, but the aforementioned experimental complications preclude physical inference based on this observation.

A mild decreasing trend can also be discerned in the measurements by Liu and Agarwal. As noted in Sect. 3.3, models based on lateral equilibration of particle motions, or small corrections thereto, reproduce this trend, but not necessarily on the basis of a physically sound representation of high-inertia deposition.

The model results in Fig. 2 correspond to a single, moderate value of $R e_{\tau}$. Sensitivity to $R e_{\tau}$ is shown in Fig. 3. There is noticeable sensitivity at moderate inertia, though not enough either to alter the physical picture or to raise concern about the comparing results for different $R e_{\tau}$ values. The only other apparent sensitivity is at very high inertia. There, the results suggest that $R e_{\tau}=180$ may be below the onset of strong-turbulence limiting behavior. The results at higher $R e_{\tau}$ show better overall conformance with the $-\frac{2}{3}$ scaling, but in contrast to the $R e_{\tau}$ dependence in (5), they indicate at most slight $R e_{\tau}$ dependence at high inertia. As noted in Sect. 3.2, this suggests that the $R e_{\tau}$ dependence in (5) is counteracted by features of turbulent channel flow that are neglected in the analysis.

Noteworthy in Figs. 2 and 3 is the falloff after the peak of $V_{d}^{+}$at a rate steeper than the $-\frac{2}{3}$ power law prior to the transition to this power-law scaling. There is no obvious explanation 
for this behavior in terms of either the model formulation or more general considerations. It would be interesting to confirm this behavior by means of a properly configured 3D simulation (see Sect. 4.4) and to perform additional analytical and computational investigations of possible new physics underlying this behavior.

Model results presented thus far are based on Stokes drag, which is inaccurate at high inertia (and in other regimes, as noted earlier). Nevertheless it is a useful idealization for elucidating physics and for comparison to simulations that use Stokes drag.

\subsection{Transient Phenomena}

Additional results are presented that focus on the transient features of high-inertia deposition. Transients are described with reference to either elapsed time (applicable to all numerical results, which are based on temporally evolving simulations, statistically homogeneous in the streamwise direction) or downstream distance (near vs. far field relative to the location of particle insertion, as in experiments). This distinction is immaterial here because quantitative comparison of numerical results and measurements is not attempted.

In Fig. 4, LES results [27] for $R e_{\tau}=180$ are plotted along with results shown in Fig. 2. The LES results exhibit decreasing $\tau_{p}^{+}$sensitivity, consistent with measurements, suggesting eventual leveling or possible decline. Also shown are model results for cases configured to be quantitatively comparable to the LES cases.

The drag law used in the LES includes the drag correction shown in (20), so it is included in the corresponding model cases. Also, for these comparisons, the particle initial conditions are the same as in the LES (uniform spatial distribution, particle velocities equal to fluid velocities). The model is run for the same wall-normalized time duration as the LES, and the sum of all particle depositions during a run is used in the determination of $V_{d}^{+}$, irrespective of possible transients, as in the LES data reduction. In the LES and in the corresponding model cases, $R e_{\tau}=180$. (Both model and LES results indicate insensitivity of $V_{d}^{+}$to $R e_{\tau}$ at the $\tau_{p}^{+}$values of interest.) For this model configuration, $V_{d}^{+}$is also shown at $\tau_{p}^{+}=50$ for comparison to the plotted DNS result at this $\tau_{p}^{+}$value. $V_{d}^{+}$is obtained using data reduction consistent with the DNS procedure, which is the same as for the LES.

Figure 4 shows that the two changes (the drag law and the time interval of data collection) relative to the baseline model results account for almost all of the discrepancy between the LES and baseline model results, and likewise improve the agreement with DNS at $\tau_{p}^{+}=50$.

Fig. 4 A portion of the data of Fig. 2, and additional results: -, transient ODT; $\triangle$, LES [27]. Otherwise, the same symbol definitions as in Fig. 2

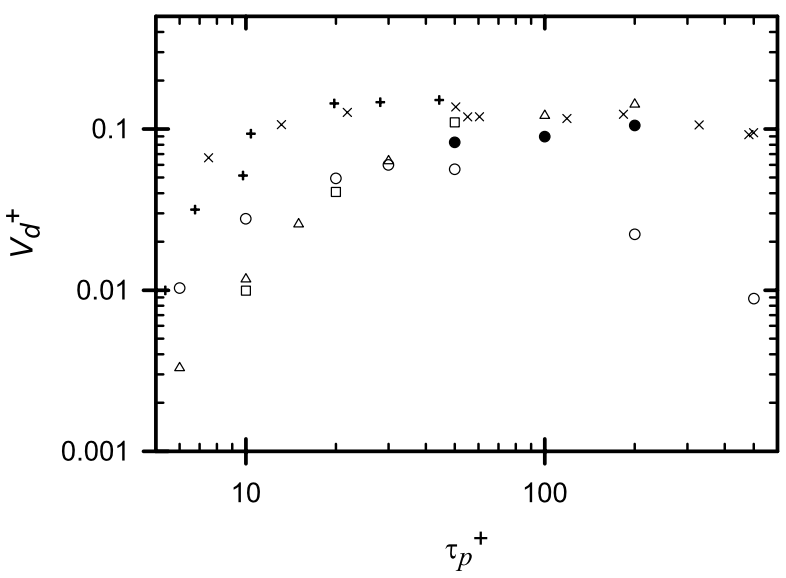




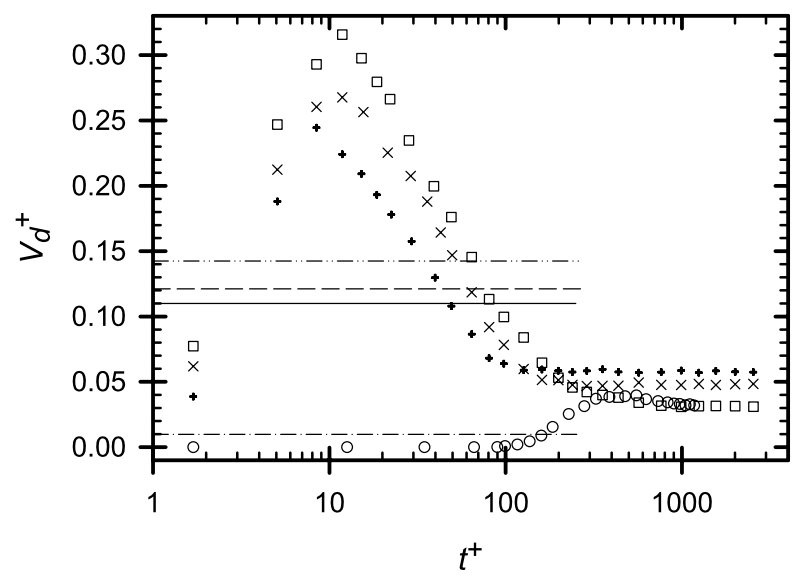

Fig. 5 For ODT at $R e_{\tau}=180$ using corrected drag, dependence of deposition rate on elapsed time $t^{+}$ in wall units for $\tau_{p}^{+}=50(+), 100(\times)$, and $200(\square)$. ०, same as the ODT $\tau_{p}^{+}=200$ case but with a zero-particle-velocity initial condition and the particle initial spatial distribution described in the text; $-\cdot-$, average for this case from $t^{+}=0$ to the end of the time interval spanned by the line. Other lines are averages that are also defined in this way, where each line extends to the ending time of the corresponding simulation: - $\longrightarrow$ DNS [1] for $\tau_{p}^{+}=50 ;---$ and $-\cdots-$, LES [27] for $\tau_{p}^{+}=100$ and 200, respectively

These observations lend credence to the predictive capability of the model based on the assigned parameter values (Sect. 4.2 and Appendix A).

The effect of the data-collection period is further elaborated in Fig. 5, in which the DNS and LES $V_{d}^{+}$values are compared to model results for time-resolved deposition. Model results indicate that the time variation of $V_{d}^{+}$during the data-collection period for each comparison case exceeds the differences between the model results and the comparison LES or DNS results that are shown in Fig. 4.

Beyond the LES data-collection period, the model results indicate relaxation to statistically steady deposition with $V_{d}^{+}$values only slightly higher than the baseline (Stokes-drag) values, indicating that the departure from baseline results that is evident in Fig. 4 is mostly due to the difference between near-field transient and far-field statistically steady deposition. For a particular initial condition (see Appendix B), Fig. 5 indicates reversal from deposition increasing to deposition decreasing with increasing inertia as the transient relaxes. The initial increase reflects the $y$ dependence of initial particle velocities.

The relaxation to statistical steadiness that is seen in Fig. 5 does not necessarily imply equilibration of particle velocities relative to turbulent motions. For large $\tau_{p}^{+}$, the analysis in Sect. 3 indicates that such equilibration does not occur during the deposition process.

Further illustrations of the distinction between transient and statistically steady deposition are provided in Figs. 5 and 6. For a variant of the $\tau_{p}^{+}=200$ model case in which particles initially have zero velocity and are uniformly distributed within a $y$ interval $[-0.6 h, 0.6 h]$, Fig. 5 shows an early period of negligible deposition followed by convergence to the $\tau_{p}^{+}=200$ model case with zero initial slip and spatially uniform initial conditions. This convergence occurs before statistically steady deposition is attained, but beyond the DNS and LES time intervals. The convergence of the two $\tau_{p}^{+}=200$ model cases is consistent with the predicted (Sect. 3.1) insensitivity to initial $U$ values provided that they are not large compared to $u^{\prime}$.

The DNS and LES studies did not report time-resolved deposition data like that shown in Fig. 5 for the ODT simulations. For comparison purposes, the single value reported for 
Fig. 6 For the ODT case denoted by + in Fig. 5, ratio of actual to ballistic (infinite-inertia) particle-deposition time, conditioned on both depositions occurring on the same wall. This ratio is shown as a function of actual deposition time in wall units

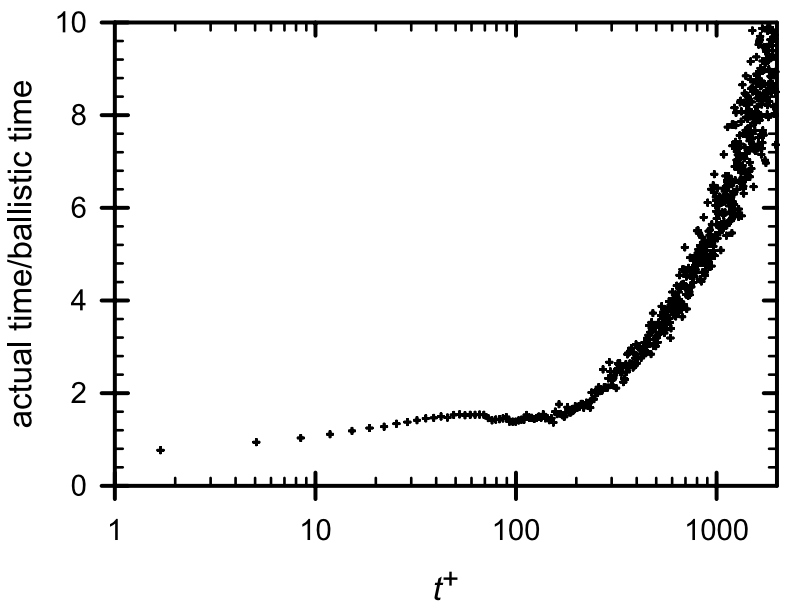

each of the 3D simulations (DNS and LES) is represented in Fig. 5 as a horizontal line that extends to the ending time of the simulation. For the zero-initial-velocity ODT case, the deposition averaged over the time interval of the LES comparison case $\left(\tau_{p}^{+}=200\right)$ is seen (lowest horizontal line) to be much lower than for the other (zero-initial-slip) cases, emphasizing that this time interval corresponds to a regime governed primarily by initial conditions.

Figure 6 shows a measure of transient relaxation for the model analog of the DNS case $\left(\tau_{p}^{+}=50\right)$. Ballistic time is the time until deposition based on a ballistic trajectory, i.e., infinite particle inertia. Inertia can increase or decrease the actual time relative to the ballistic time, but a net decrease occurs only at very early times when deposition is promoted by 'favorable' eddy motions acting on particles initially near a wall. (To simplify the interpretation of this metric, particles that deposit on the wall opposite to the wall that would be encountered ballistically are disregarded.) The relatively low ratio of actual to ballistic time prior to $t^{+}=150$, corresponding to 3 particle time constants, reflects the deposition of particles whose trajectories closely conform to the initial orientation of the particle velocity vector. The subsequent rapid growth and increased scatter of this ratio reflects the transition to deposition governed primarily by deviation from the initial direction of particle motion. Figure 5 indicates that this onset coincides with the attainment of statistically steady deposition for this case. In contrast, the apparent convergence of the two $\tau_{p}^{+}=200$ cases in Fig. 5 prior to their attainment of statistical steadiness suggests that memory loss precedes the completion of transient relaxation. The overlap of these cases during the late transient is imperfect and may be fortuitous; they unambiguously coincide only when statistical steadiness is attained.

Further detailed investigation would be required to form a complete picture of the process of transition from initial condition sensitivity to insensitivity and the related but distinct process of transient relaxation. The foregoing observations are suggestive of the rich phenomenology of non-equilibrium deposition that remains to be explored. This phenomenology should be explored using a variety of methods because no single method, including those introduced here, is both fully accurate and applicable to all regimes of interest. 


\section{Discussion}

Although the possible influence of non-equilibration of particle-flow interactions on deposition from turbulent flow is suggested by studies to date, its role in the high-inertia regime has not been probed sufficiently to discern the underlying physics. The scaling analysis presented here predicts $-\frac{2}{3}$ power-law decay of the deposition rate as a function of particle Stokes number $\tau_{p}^{+}$for large $\tau_{p}^{+}$and indicates that high-inertia deposition is a nonequilibrium process. A stochastic model that captures the relevant physics of particle-flow coupling and simulates turbulent channel flow accurately, yet is economical enough for simulation of deposition at very high $\tau_{p}^{+}$and determination of time-resolved deposition rates, is formulated and applied to channel-flow deposition over a wide range of $\tau_{p}^{+}$. The model reproduces much of the phenomenology of channel-flow deposition and elucidates the features of the high-inertia regime.

Specifically, is it shown that the predicted scaling is a far-field (or long-time, in temporal flow) asymptote preceded by transient relaxation during which deposition is nearly ballistic and therefore not very sensitive to $\tau_{p}^{+}$. The deposition rate during this transient may exceed the rate during the subsequent statistically steady deposition, so a majority of particles may deposit during the transient. Typically, the particles that survive the transient are those whose initial trajectories are nearly streamwise so that ballistic motion does not cause early deposition.

The relative contributions of transient and far-field deposition are sensitive to initial conditions. Particles with negligible initial lateral velocity are not subject to the ballistic mechanism, so the deposition rate for particles initialized in this matter is lower during the transient than subsequently.

Except for the atypical situation in which the particles are highly collimated initially, almost all particles deposit during the transient phase. Thus, far-field deposition is generally not of practical importance, unless one is concerned about particles not deposited rather than particles deposited. For example, if wall deposition is used as a mechanism for removal of hazardous or otherwise undesirable particulate material from a turbulent fluid stream, very low outflow particle concentration might be required. Modeling that does not account for far-field non-equilibrium behavior could grossly underestimate the outflow particle concentration, and therefore could provide misleading design guidance.

It is emphasized here that statistically steady deposition does not imply equilibration of particle motions relative to turbulent fluid motions. In fact, it is found that the particle motions are far from equilibrium in the statistically steady deposition regime. An additional subtlety that deserves further attention is the observation that the disappearance of sensitivity to initial conditions may occur before deposition becomes statistically steady.

The results presented here suggest a sequence of as many as four types of behavior as a function of elapsed time during high-inertia deposition: (1) transient and sensitive to initial conditions, (2) transient, but insensitive to initial conditions, (3) statistically steady, but nonequilibrium, and (4) the notional equilibrium regime, postulated in closure models but shown here to be unattainable, except possibly during rare events involving particles that remain in the flow for exceptionally long times (a scenario that has not been investigated here). In addition to this rich phenomenology in the time domain for given $\tau_{p}^{+}$, numerical results indicate an as-yet-unexplained rapid decline of the deposition rate as a function of $\tau_{p}^{+}$prior to the onset of the high-inertia scaling whose physical basis has been identified through scaling analysis. These observations suggest several avenues of future investigation.

In previously reported 3D numerical simulations of deposition in the vicinity of the onset of the inertia-moderated regime, the initial particle slip velocity is set equal to zero, 
a scenario in which the transient deposition rate exceeds the far-field rate and the transient deposition exhibits nearly ballistic scaling (insensitivity to $\tau_{p}^{+}$). These simulations are costly, which may be the reason they were not run long enough to reach the far-field regime beyond the initial transient, nor replicated to obtain enough data for time-resolved determination of $V_{d}^{+}$, whose importance has been demonstrated here.

The increase of computer capabilities subsequent to these 3D simulation studies provides the wherewithal for future $3 \mathrm{D}$ numerical exploration of the time domain as well as a relevant range of $\tau_{p}^{+}$values. The model results presented here may provide useful guidance for planning these studies and interpreting the results. Independent verification of the results presented here is desirable due to the simplifications inherent in both the scaling analysis and the computational model. 3D simulation is a suitable method for obtaining this verification.

Experimental investigation of time-resolved (more precisely, streamwise-resolved because the flow development in experiments is spatial rather than temporal) high-inertia deposition is more problematic. It is difficult to design an experiment in a confined flow (e.g., a pipe) that is long enough to allow transient relaxation yet is not subject to complications that obscure the quantitative signatures of convergence to statistically steady deposition. One implication of the present study with regard to measurements is that the well-known inconsistencies among various reported measurements of high-inertia deposition $[16,28]$ can perhaps be attributed to the sensitivity to initial conditions during the near-field transient, although other contributing factors may be equally if not more significant. The present study implies that collimation of injected particles or droplets would tend to prevent the obscuration of statistically steady deposition by the initial transient.

The results presented here indicate that the measurements and numerical results heretofore presumed to confirm closure-model predictions of high-inertia deposition are subject to artifacts that cast doubt on the claimed confirmation. In fact, it appears that the closure models do not capture the leading-order non-equilibrium behavior resulting from the time-lagged response of particles to turbulent fluid motion, and the consequent deposition phenomenology. Thus, concise modeling of high-inertia deposition (and by implication, high-inertia behavior in general) is a greater challenge than is commonly supposed. One goal of the present study is to encourage further progress in this regard by identifying some of the physics and phenomenology that models need to capture.

Graham [5] addresses initial-condition sensitivity, slow relaxation of transients, nonequilibrium effects, and implications concerning the design and interpretation of experiments and numerical simulations, in the context of particle dispersion in isotropic turbulence. This context allows more detailed analysis than is presented in Sect. 3. The present contribution may be viewed as a demonstration, using heuristic analysis and modeling, of the impact of these considerations in the context of deposition in confined turbulent flow.

Thus, the issues addressed here are broader than this particular application. It is anticipated that future application of the analytical and numerical modeling approaches introduced here to other multiphase turbulent flow regimes might provide further useful insight. In this regard, generalization of the present two-phase-flow formulation of ODT to include more elaborate drag laws and multiple scalars, allowing inter-phase couplings such as heat and mass transfer and thermophoresis, should be possible. The modeling framework also accommodates other generalizations such as two-way coupling. With these generalizations, ODT will be complementary to other particle-eddy-interaction models [7]. Other models of this type might be applicable to some of the issues considered here, but they generally lack the spatial and temporal resolution of microphysical details needed for accurate representation of various molecular-transport contributions to inter-phase couplings. 
Acknowledgements Portions of this work were completed by the first author (1) as his Ph.D. Dissertation in Chemical Engineering at the University of Arizona, and (2) during a National Research Council (NRC) Research Associateship at the Army Research Laboratory (ARL). This work was also partially supported by the US Department of Energy, Office of Basic Energy Sciences, Division of Chemical Sciences, Geosciences, and Energy Biosciences. Sandia National Laboratories is a multi-program laboratory operated by Sandia Corporation, a Lockheed Martin Company, for the United States Department of Energy under contract DEAC04-94-AL85000.

Open Access This article is distributed under the terms of the Creative Commons Attribution Noncommercial License which permits any noncommercial use, distribution, and reproduction in any medium, provided the original author(s) and source are credited.

\section{Appendix A: ODT Simulation of Channel Flow}

An ODT formulation previously used to simulate turbulent channel flow [21] is adopted in the present study. Because it is described and validated in the cited reference and other published work [14], the description here is brief.

It is instructive to introduce for comparison a simple boundary-layer representation of fully developed channel flow, statistically stationary and statistically homogeneous in the streamwise $(x)$ direction, based on eddy-viscosity modeling,

$$
\partial_{t} u=\partial_{y}\left(v_{e}+v\right) \partial_{y} u-\left(1 / \rho_{f}\right) \partial_{x} p,
$$

where $t$ is time (denoted $t_{f}$ in Appendix B to distinguish it from a particle time coordinate), $y$ is the lateral (wall-normal) coordinate, $u(y, t)$ is the streamwise velocity, $v$ is kinematic viscosity, $v_{e}$ is the modeled eddy viscosity, $\rho_{f}$ is density (assumed constant), and $\partial_{x} p$ is an imposed mean pressure gradient. $v_{e}$ is typically assumed to be a time-invariant function of $y$, in which case (7) has a steady-state solution $u(y)$ representing the mean flow velocity.

The eddy viscosity in (7) is a surrogate for advective transport of $u$ by lateral velocity fluctuations. There is no $v \partial_{y} u$ term representing transport by the mean lateral velocity $v$ because $v$ is identically zero under the stated assumptions.

For affordable simulation of high-intensity turbulence, a 1D formulation is sought that likewise involves a surrogate representation of advective transport, but that captures both the unsteady time evolution and the fine-grained spatial structure of the flow. These features enable a detailed representation of particle-flow interaction, as described in Appendix B. The adoption of a time-developing formulation based on streamwise statistical homogeneity is consistent with 3D numerical formulations to which the model is compared in Sect. 4.

The surrogate representation that is adopted models unsteady lateral advection by means of a stochastic sequence of maps applied to the $y$ coordinate, such that the sequence of induced lateral displacements emulates the statistical properties of turbulent advection while obeying the applicable conservation laws. The sampling of maps, though stochastic, is dependent on the instantaneous flow state in a manner that introduces a dynamic linkage between past and future map occurrences. Through this linkage, the model captures key turbulence phenomena such as the inertial-range cascade.

Formally, the outcome of simulated evolution from time 0 to time $t$ can be expressed as

$$
\begin{aligned}
\mathbf{u}(y, t)= & T\left[t_{n}, t\right] E\left(\mathbf{p}_{n}\right) \cdots T\left[t_{j}, t_{j+1}\right] E\left(\mathbf{p}_{j}\right) T\left[t_{j-1}, t_{j}\right] \\
& \cdots E\left(\mathbf{p}_{2}\right) T\left[t_{1}, t_{2}\right] E\left(\mathbf{p}_{1}\right) T\left[0, t_{1}\right] \mathbf{u}(y, 0),
\end{aligned}
$$


where capital letters represent operators. $T\left[t_{j-1}, t_{j}\right]$ represents time advancement of the operand $\mathbf{u}\left(y, t_{j-1}\right)$ from $t_{j-1}$ to $t_{j}$, governed by

$$
\partial_{t} u_{i}=\partial_{y} v \partial_{y} u_{i}-\left(\delta_{i, 1} / \rho_{f}\right) \partial_{x} p
$$

with boundary conditions $\mathbf{u}(y, t)=0$ at $y= \pm h$. Equation (9) is analogous to (7) but includes the full velocity vector $\mathbf{u}=\left(u_{1}, u_{2}, u_{3}\right)$ and omits the eddy-viscosity term. ( $u_{i}$ velocity-component notation is used only in this Appendix.) In place of eddy viscosity, lateral turbulent advection is represented by 'eddy events' $E\left(\mathbf{p}_{j}\right)$, each of which is an instantaneous transformation of the operand $\mathbf{u}\left(y, t_{j}\right)$ representing a turbulent eddy motion, where $\mathbf{p}_{j}$ is the set (vector) of randomly sampled quantities that parameterize the $j$ th transformation. $n$ is the number of eddy events during $[0, t], t_{j}$ is the time of occurrence of the $j$ th event for $j>0$, and $t_{0}=0$. The epochs $t_{j}$, the quantities $\mathbf{p}_{j}$, and $n$ are random variables evaluated during each simulated realization by a sampling procedure whose rules embody the principal physical content of the model. Event occurrences are sampled from an event rate distribution, (14), that is based on a time scale $\tau(\mathbf{p} ; t)$, analogous to an eddy turnover time, associated with each possible event $\mathbf{p}$ and evaluated as a function of the entire flow state at time $t$.

Each eddy event consists of two mathematical operations. One is a measure-preserving map representing the fluid displacements. The other is a modification of the velocity profiles that redistributes energy while conserving momentum. The combined effect of these operations is denoted

$$
u_{i}(y) \rightarrow u_{i}(f(y))+c_{i} K(y) .
$$

The fluid at location $f(y)$ is moved to location $y$ by the mapping operation, denoted the 'triplet map' (see below). This mapping is applied to all fluid properties, including scalars in variable-property flows (not considered here). The additional term $c_{i} K(y)$, which is only applied to the velocity components, is the ODT analog of pressure-induced kinetic-energy redistribution among the velocity components, and also accommodates energy exchange with sources and sinks such as gravitational potential energy in buoyant-flow applications.

The eddy parameters $\mathbf{p}$ are a lower eddy boundary $y_{0}$ and a size $l$, determining the upper boundary $y_{0}+l$. The triplet map maps the interval $\left[y_{0}, y_{0}+l\right]$ onto each of three images that occupy successive thirds of the interval, thus filling the interval. Each image is a threefold compression of the original interval, except that the central (second) image is also inverted (spatially flipped). The map from location $f(y)$ to location $y$ is expressed formally as

$$
f(y) \equiv y_{0}+ \begin{cases}3\left(y-y_{0}\right) & \text { if } y_{0} \leq y \leq y_{0}+\frac{1}{3} l, \\ 2 l-3\left(y-y_{0}\right) & \text { if } y_{0}+\frac{1}{3} l \leq y \leq y_{0}+\frac{2}{3} l, \\ 3\left(y-y_{0}\right)-2 l & \text { if } y_{0}+\frac{2}{3} l \leq y \leq y_{0}+l, \\ y-y_{0} & \text { otherwise. }\end{cases}
$$

Graphical illustrations and extensive discussions of the triplet map are presented elsewhere $[10,11]$. Recently, it was independently reintroduced and used for analysis of the turbulent cascade [9].

In the energy-redistribution term of (10), $K(y)$ denotes $y-f(y)$, the displacement of the fluid element mapped to $y$. It is non-zero only within the eddy interval $\left[y_{0}, y_{0}+l\right]$ and it integrates to zero so that the eddy event does not change the total ( $y$-integrated) momentum of individual velocity components. 
The remaining ingredient in the specification of the eddy event is the expression for $c_{i}$,

$$
c_{i}=\frac{27}{4 l}\left[-u_{i, K}+\operatorname{sgn}\left(u_{i, K}\right) \sqrt{\frac{1}{3}\left(u_{1, K}^{2}+u_{2, K}^{2}+u_{3, K}^{2}\right)}\right],
$$

where

$$
u_{i, K}=l^{-2} \int u_{i}(f(y)) K(y) d y .
$$

This is obtained by requiring that the 'available kinetic energy' of the post-map profiles of the respective velocity components should be the same for all velocity components (equalization of available kinetic energy, the ODT analog of 'return to isotropy' terms in closure models). The available kinetic energy of component $u_{i}$ is determined by finding the value of $b_{i}$ that minimizes the kinetic energy of the profile $u_{i}+b_{i} K(y)$. The difference between the kinetic energies of the $u_{i}$ and $u_{i}+b_{i} K(y)$ profiles is the available kinetic energy.

Eddy sampling is based on the eddy rate distribution

$$
\lambda\left(y_{0}, l ; t\right)=\frac{C}{l^{2} \tau\left(y_{0}, l ; t\right)}=\frac{C v}{l^{4}} \sqrt{\frac{1}{3}\left[\left(\frac{u_{1, K} l}{v}\right)^{2}+\left(\frac{u_{2, K} l}{v}\right)^{2}+\left(\frac{u_{3, K} l}{v}\right)^{2}\right]-Z}
$$

where $\lambda\left(y_{0}, l ; t\right) d y_{0} d l d t$ is the probability of an event with parameters in the ranges $\left[y_{0}, y_{0}+d y_{0}\right]$ and $[l, l+d l]$, respectively, during $[t, t+d t]$. If the radical in (14) has a negative argument, the event rate is taken to be zero. Physically, this represents viscous suppression of the eddy, governed by the parameter $Z$. $C$ (which scales the rate distribution) and $Z$ are two adjustable parameters of the present ODT formulation. Following [21], they are assigned the values $C=12.73$ and $Z=98$, and the constraint $l<h$ (a third empirical assignment) is applied. Incorporation of inertial particles introduces one additional adjustable parameter (see Appendix B).

Equation (14) embodies the core physical content of ODT. Its derivation and features are discussed extensively in publications $[12,14]$ and in web-accessible reference material, including a documented code, ${ }^{1}$ so they are not explained here. Efficient event sampling based on (14) is done by 'thinning,' a generalization of the von Neumann rejection method.

\section{Appendix B: Inertial-particle Submodel}

\section{B.1 Particle Response to Turbulent Motion}

Because ODT evolves all three velocity components, an obvious way to incorporate inertial particles is to evolve particle velocities $U, V$, and $W$ by substituting the ODT-specified fluid velocities $u(Y, t), v(Y, t)$, and $w(Y, t)$, into the particle drag law, where $Y$ is the particle lateral location at time $t$. Time integration of $U, V$, and $W$ advances the particle location $(X, Y, Z)$. Because the particle is confined to the ODT domain, the ODT fluid state is deemed to represent the lateral profiles of the fluid velocity components at the $(X, Z)$ location at which the particle currently resides. Flow time advancement on the ODT domain thus represents the spatial as well as temporal variability of the fluid velocity seen by the

\footnotetext{
${ }^{1}$ http://groups.google.com/group/odt-research
} 
particle. This implies a relationship between particle and flow advancement that is explained in Sect. B.2.

This approach has been implemented and results have been compared to measurements and simulations of various particle statistics in turbulent channel flow [23]. Although this approach is found to be successful in some respects, it is not pursued here because it violates a key property of particle advection: in the zero-inertia (marker-particle) limit, a particle should remain within the fluid element that contains it initially. To obey this requirement, the particle-fluid coupling must allow instantaneous displacement of particles by eddy events. Because this coupling is based on a drag law that must be time integrated to obtain its effect on particle displacement, it is evident that some method must be devised to account for the finite time duration of turbulent motions. The method used here is outlined below and further elaborated in [20].

The needed ingredients are available within ODT. The specific goal is to interpret a mapinduced fluid displacement $y \rightarrow y^{\prime}$ (different notation than in Appendix A) as fluid motion at some specified velocity $v_{E}$ that is constant (for simplicity) for some specified time interval $t_{E}$ that is here termed the eddy lifetime. In Appendix A, it is noted that eddy event selection involves the specification of an eddy time scale $\tau$. Because this is the only time scale available in ODT to represent eddy properties, the eddy lifetime $t_{E}$ is modeled as $\beta \tau$, where $\beta$ is an adjustable parameter, the only new parameter required in the ODT particle representation. To obtain the specified lateral displacement from motion at constant lateral velocity during the time interval $t_{E}$, the velocity must obey

$$
v_{E}=\left(y^{\prime}-y\right) / t_{E} .
$$

It is now straightforward in principle to specify the particle-eddy interaction, although some details require care. The present approach has much in common with particle-eddyinteraction sub-models that have been used in steady-state-flow formulations [6, 7], although the implementation is different in several respects. To keep the development general, it is not specialized to a particular form of the drag law until Sect. B.3.

Assume that a particle is located at $(X, Y, Z)$ and has velocity components $(U, V, W)$ at the time $t_{j}$ when the $j$ th eddy event occurs and assume that $Y$ is within the eddy interval $\left[y_{0}, y_{0}+l\right]$. Then the particle location $Y$ is mapped by the eddy event to each of three locations $y_{k}^{\prime} \cdot y^{\prime}$ in (15) is evaluated by randomly sampling one of the locations $y_{k}^{\prime}$, with probability $\frac{1}{3}$ of choosing each. Each sampling is independent for each particle within the eddy event and for each eddy event encountered by a particle during the simulation. It has been shown that this procedure yields a physically sound representation of the evolution of a particle ensemble in turbulence [13].

Note that while $y^{\prime}$ is the location to which fluid at $Y$ is mapped, the particle is not necessarily mapped to $y^{\prime}$ if it has nonzero inertia. The procedure determining the final particle location $Y^{\prime}$, as well as the other descriptors of the particle state at the conclusion of the particle-eddy interaction, is now considered.

During the particle-eddy interaction, whose time duration is denoted $t_{I}$, the lateral velocity of the fluid initially at $y=Y$ is modeled according to (15). It is not strictly accurate to infer that the particle initially at $y=Y$ is subject to this fluid velocity throughout the interaction period because the trajectory of an inertial particle will deviate from the trajectory of the fluid initially at $Y$. Nevertheless, the modeling assumption is adopted that $v_{E}$ is the fluid velocity seen by the particle throughout the interaction period. Alternate assumptions introduce additional complexity with no clear gain in overall physical fidelity because the particle-eddy coupling is in any case an idealization. 
The duration of the interaction is denoted $t_{I}$ rather than $t_{E}$ because the particle may cease to interact with the eddy before the eddy reaches the end of its life. This cessation is due to the emergence of the particle from the spatial region occupied by the eddy. In ODT, the $y$ interval occupied by the eddy, denoted $\left[y_{0}, y_{0}+l\right]$, is specified by the eddy sampling procedure. Taking the eddy to be a cube (solely for the purpose of evaluating $t_{I}$ ), its streamwise and spanwise extent relative to the particle location are both taken to be $[-l / 2, l / 2]$. The cube is advected in the streamwise direction at a velocity equal to the average of $u(y, t)$ over $y_{0} \leq y \leq y_{0}+l$. These geometrical properties, in conjunction with the particle trajectory solution described shortly, determine the first occurrence, if any, of particle emergence from the spatial region occupied by the eddy. If this occurs before elapsed time $t_{E}$, then $t_{I}$ is set equal to the time of this first occurrence, otherwise $t_{I}$ is set equal to $t_{E}$. Accounting for the distinction between $t_{I}$ and $t_{E}$ is important when particle-eddy interaction is dominated by trajectory-crossing effects (see Sect. B.2).

Within this framework, the particle drag law is time integrated, based on the fluid velocity modeled as $\left(u\left(Y, t_{j}\right), v_{E}, w\left(Y, t_{j}\right)\right)$, to advance the particle from its state $(X, Y, Z, U, V, W)$ at time $t_{j}$ to its state $\left(X^{\prime}, Y^{\prime}, Z^{\prime}, U^{\prime}, V^{\prime}, W^{\prime}\right)$ at time $t_{j}+t_{I}$, where this advancement contributes to the determination of $t_{I}$ as explained above. For consistency, the streamwise and spanwise, as well as lateral, fluid velocity components are evaluated at the initial (time $t_{j}$ ) particle location.

Although this advancement specifies new values of all particle coordinates and velocity components, only the lateral particle location and velocity are modified by the particleeddy interaction. This is because evolution of the other coordinates and velocity components are adequately represented by particle advancement during the time intervals between eddy events. (Recall that the special treatment of lateral advancement is for the specific purpose of assuring that eddy events result in appropriate marker-particle behavior in the zero-inertia limit.) Thus, the updated state variables $\left(X^{\prime}, Z^{\prime}, U^{\prime}, W^{\prime}\right)$ obtained from drag-law integration play no role in subsequent advancement. These variables are time advanced during particleeddy interaction for the sole purpose of evaluating $t_{I}$ by following the particle trajectory to determine whether emergence from the eddy occurs during the eddy lifetime, and if so, when.

The provisional particle state (subject to a modification described shortly) at the end of particle-eddy interaction is thus $\left(X, Y^{\prime}, Z, U, V^{\prime}, W\right)$. The primed variables reflect advancement for a time interval $t_{I}$ starting from the state at time $t_{j}$ and the unprimed variables correspond to the state at $t_{j}$.

ODT eddies are instantaneous, so the state immediately after eddy $j$ corresponds to time $t_{j}$. Time advancement subsequent to particle-eddy interaction (particle $T$ process; see below) thus begins at time $t_{j}$, implying a period of advancement in physical time that overlaps the time advancement from $t_{j}$ to $t_{j}+t_{I}$ that is implemented during particle-eddy interaction.

The duplication of particle advancement implied by this overlap is corrected as follows. Particle-eddy interaction as described thus far determines new values $\left(Y^{\prime}, V^{\prime}\right)$ of the particle lateral location and velocity by integrating the drag law for a time interval $t_{I}$ starting from the initial state $(Y, V)$ and holding the fluid velocity seen by the particle fixed at $\left(u\left(Y, t_{j}\right), v_{E}, w\left(Y, t_{j}\right)\right)$. A second drag-law integration is introduced, over the same time interval and starting from the same initial state, except that the fluid velocity seen by the particle is held fixed at $\left(u\left(Y, t_{j}\right), 0, w\left(Y, t_{j}\right)\right)$. The values of the particle lateral variables upon completion of this integration are denoted $\left(Y_{0}^{\prime}, V_{0}^{\prime}\right)$. The physical state of the particle upon completion of particle-eddy interaction is then taken to be $\left(X, Y^{\prime \prime}, Z, U, V^{\prime \prime}, W\right)$, where $Y^{\prime \prime}=Y+Y^{\prime}-Y_{0}^{\prime}$ and $V^{\prime \prime}=V+V^{\prime}-V_{0}^{\prime}$. 


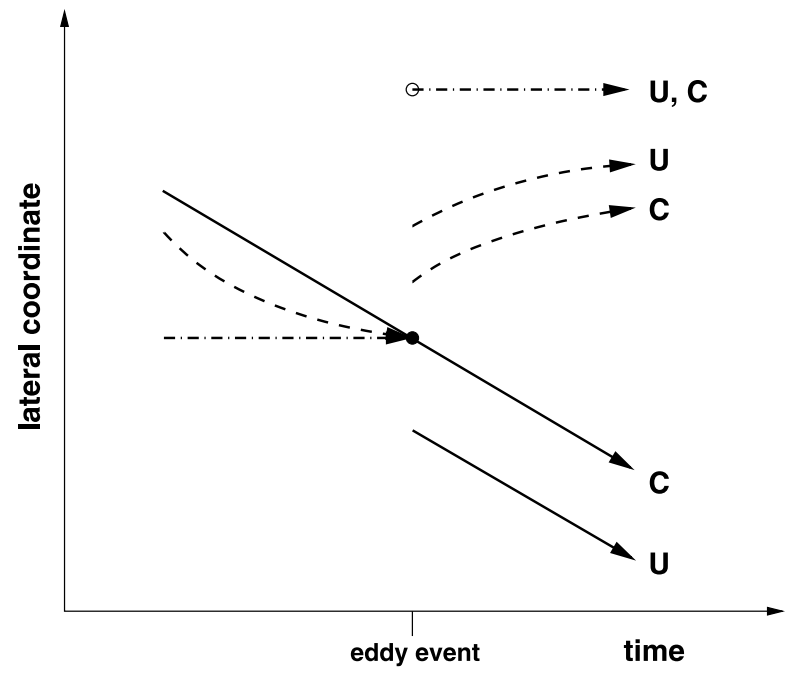

Fig. 7 Schematic illustration of particle-eddy interaction for particles whose inertia is zero (- - -), finite (- - - ), and infinite (- ${ }_{-}$. •, location of fluid element containing the particles at the time of an eddy event, but prior to fluid displacement; o, location of that fluid element after eddy-induced displacement; U (uncorrected), notional particle trajectory omitting the correction; $\mathrm{C}$ (corrected), actual particle trajectory incorporating the correction. For zero inertia, the correction vanishes, so trajectories $\mathrm{U}$ and $\mathrm{C}$ are identical and coincide with the trajectory of the fluid element. For infinite inertia, trajectory $U$ is discontinuous because the particle-eddy interaction advances the particle drag law for a finite time interval but the physical time is not incremented. The correction exactly compensates for this, so trajectory $\mathrm{C}$ is ballistic. For finite inertia, trajectories $\mathrm{U}$ and $\mathrm{C}$ are both discontinuous and neither coincides with the fluid-element trajectory

The rationale for this procedure is illustrated by considering the limiting cases of infiniteinertia and zero-inertia particles. For infinite particle inertia, the value of $v_{E}$ has no effect on particle advancement, so $Y^{\prime}=Y_{0}^{\prime}$, giving $Y^{\prime \prime}=Y$. Likewise, $V^{\prime \prime}=V$. Thus, the eddy interaction has no effect on the particle and it continues on its ballistic (linear) trajectory during subsequent advancement. (The particle $T$ process governing time advancement between eddy interactions is explained shortly.) This is the physically correct result. For zero particle inertia, $Y^{\prime}=y^{\prime}, Y_{0}^{\prime}=Y$, and thus $Y^{\prime \prime}=y^{\prime}$, which is the physically correct markerparticle behavior. The result for $V^{\prime \prime}$ is immaterial because the marker particle immediately relaxes to zero velocity when the subsequent time advancement commences.

It is seen that the subtraction procedure is suitable for the limiting cases, and representative case studies show that it provides a reasonable interpolation between these cases for finite-inertia particles [20]. Conceptually, the procedure evaluates the net effect of the interaction on the particle relative to particle evolution if the eddy had not occurred. (See the graphical illustration in Fig. 7; additional illustrations and explanation are provided in [20].) By defining the particle-eddy interaction based on this difference, it is consistent to implement the outcome of the interaction instantaneously at time $t_{j}$ despite the fact that it is computed by time integration from $t_{j}$ to $t_{j}+t_{I}$.

For some configurations, e.g., particles moving rapidly in the ODT domain direction $y$, the instantaneous nature of eddy events might cause particles to encounter eddy events much less often than they would encounter physical eddies of finite time duration. Accurate modeling of such configurations requires eddy events as perceived by particles to have finite durations in the physical time coordinate as well as in the internal time coordinate introduced to model particle-eddy interactions. A formulation of this type has been developed [20] but 
it is not used here because it is considerably more elaborate than the present formulation, which is suitable for the flow regimes considered here.

The evolution of a particle within an ODT simulation can be represented schematically by (8), but with different interpretation of symbols than for fluid evolution. In (8), $\mathbf{u}$ is replaced by the particle state $(X, Y, Z, U, V, W)$, evaluated at time $t$ on the left-hand side and at time 0 on the right-hand side. $E$ is now interpreted as a particle-eddy interaction. Description of the model is now completed by specifying the particle advancement $T$ that occurs during the time intervals between these interactions.

During the particle $T$ process, the particle state $(X, Y, Z, U, V, W)$ at time $t$ is time advanced based on the drag law with fluid velocity $(u[Y(t), t], 0, w[Y(t), t])$, where the lateral component of fluid velocity is set equal to zero in order to maintain consistency with the marker-particle limit. In ODT as implemented here (Appendix A), fluid is advected laterally only by eddy events. Hence, there is no $v \partial_{y} u_{i}$ term in (9); the ODT $v$ velocity profile is an auxiliary variable that does not directly govern lateral fluid advection.

One consequence of this formulation is that $V$ converges toward zero during the $T$ process (and vanishes instantly for zero inertia). This is an artifact of the separation of the time coordinate into finite intervals with zero lateral motion, alternating with instantaneous lateral displacements. This can result in unrealistically low particle $V$ values upon the initiation of particle-eddy interactions. Therefore the initial $V$ value in the time integration performed to evaluate the particle-eddy interaction is taken to be not the current value, but the value $V^{\prime \prime}$ obtained as the outcome of the most recent eddy interaction involving the given particle. (As noted, $V$ is nevertheless time advanced during the $T$ process so that the particle trajectory $Y(t)$ obeys physical requirements such as correct behavior in the marker-particle limit.)

\section{B.2 Representation of Trajectory-crossing Effects}

Trajectory-crossing effects are statistical biases of flow time histories seen by particles due to their motion relative to the fluid. In Sect. 3.1, the estimate of $t_{c}$ for $U_{0} \gg u^{\prime}$ models these effects. Through the evaluation of $t_{I}$, the ODT particle-eddy interaction has been formulated to take account of trajectory-crossing effects.

Trajectory-crossing effects are likewise incorporated into the $T$ process. Explicit treatment of these effects during the $T$ process is needed because the particle streamwise advancement $d X=U d t$ implies trajectory crossing if $d X \neq d x$ where $d x$ is the fluid streamwise advancement $u(Y, t) d t$ at the particle location. Particle advancement from $t$ to $t+d t$ subject to drag influence should reflect both flow temporal evolution from $t$ to $t+d t$ and particle traversal of a streamwise interval $d X-d x$ of the flow. The latter contribution is not explicit in ODT because the 1D domain represents a flow profile co-moving with the particle in $x$ (and in $z$ ), so $d x=d X$.

Therefore the additional contribution to flow temporal variability seen by a particle due to the difference between flow states separated by the streamwise distance $d X-d x$ is modeled by time advancing the flow more rapidly than the particle. Namely, $u_{b}$ is taken to be the velocity scale relating spatial and temporal variability through the relation $d x=u_{b} d t_{f}$, where $d t_{f}$ is the flow time increment. This means that the velocity change of a fluid element during $d t_{f}$ is assumed to be the same as the velocity difference between two points separated by a distance $u_{b} d t_{f}$ at a given instant. (The choices $d t_{f}=T$ and $d x=h$ show the plausibility of this assumption.) To model this effect, $d t_{f}$ is increased relative to $d t$ by an amount $|d X-d x| / u_{b}$. Substitution of $d X=U(t) d t$ and $d x=u(Y, t) d t$ gives

$$
d t_{f}=\left[1-\frac{|U(t)-u(Y, t)|}{u_{b}}\right] d t .
$$


This model is subject to verification, but present results do not test this model because its effect is minor relative to other model assumptions for the cases discussed in Sect. 4. Future applications of this formulation to cases involving more consequential streamwise trajectory-crossing effects, such as the regime $U_{0} \gg u^{\prime}$ considered in Sect. 3.1, will test (16) and possibly motivate improvements, such as substituting a $y$-dependent velocity scale for $u_{b}$ in order to improve the representation of trajectory-crossing effects near walls.

\section{B.3 Simulation of Deposition in Channel Flow}

Because the particles do not affect the flow, $t_{f}$ can be used as the time variable for simulation advancement, with (16) then specifying a distinct time variable $t$ for the advancement of each particle. Asynchronous particle advancement implies limited applicability of this formulation to multi-particle phenomena such as clustering, for which a variant of the present approach is more suitable [13].

On this basis, turbulent-channel-flow simulation is implemented as in [21] using the same ODT parameter values as in that study (see Appendix A). The flow configuration is fully specified by the quantity $R e_{\tau}$.

Before particles are introduced, the flow simulation is run until it is statistically steady. At a given instant, particles are introduced. For the cases shown in Figs. 1, 4, 5, and 6, the initial velocity of each particle is chosen to match the local fluid velocity unless stated otherwise. For the cases shown in Figs. 2 and 3, a method proposed by Graham [5] is used to assign the initial velocity of each particle. (For steady-state deposition, initialization affects the speed of transient relaxation but not the steady-state behavior.) The initial lateral distribution of particles is spatially uniform unless stated otherwise.

Specifically, the flow evolves on a uniform mesh, and one particle is introduced in the center of each mesh cell. The coarsest meshes have order $10^{3}$ cells and the finest mesh has order $10^{4}$ cells. Grid insensitivity was verified by repeating simulations with double the number of grid points and ensuring that the steady-state $V_{d}^{+}$value does not change to two significant figures.

The precision is dependent on the run time during statistically steady conditions. Efficient utilization of computer resources mandates ending a given realization when the remaining number of particles gets small compared to the initial number of particles. The desired precision is then obtained by running multiple realizations in order to accumulate the needed total run time, as determined by the needed number of particle depositions. Not all deposition events are included in the evaluation of $V_{d}^{+}$. Time-resolved deposition statistics, discussed in Sect. 4.4, indicate transient relaxation that is especially significant for large $\tau_{p}^{+}$. Therefore $V_{d}^{+}$is evaluated on the basis of depositions during the period of statistically steady deposition following transient relaxation (or for the transient results in Fig. 5, within individual time bins that resolve the transient).

For steady-state deposition, the number of simulated realizations and run time per realization were chosen for various cases so that the number of particle depositions per plotted $V_{d}^{+}$value ranged from a minimum of order $10^{2}$ at low inertia to order $10^{6}$ in the vicinity of $\tau_{p}^{+}=100$, then declining to order $10^{4}$ at high inertia. In the $\log -\log$ plots of $V_{d}^{+}$versus $\tau_{p}^{+}$ that are shown in Sect. 4, the uncertainty of the results is in all instances smaller than the symbol size.

Particles are assumed to be spherical. When a particle reaches a lateral location $Y$ that is within one radius of a wall, it is deemed to deposit on the wall and disappears from the simulation. Although particle radii are finite for consistency with comparison cases, they are small enough in all instances so that finite-size effects (relative to the limit of zero radius and 
infinite density such that $\tau_{p}^{+}$is fixed) are negligible. Ghost particles are assumed, i.e., there is no particle-particle interaction, so the simulation can be run at arbitrary particle volume fraction and still represent dilute conditions.

The model can accommodate drag laws of various degrees of complexity. Here, particle motion is governed by

$$
\begin{aligned}
\frac{d \mathbf{S}}{d t} & =(\mathbf{s}-\mathbf{S}) \frac{f}{\tau_{p}}, \\
\frac{d \mathbf{X}}{d t} & =\mathbf{S}, \\
\tau_{p} & =\frac{2}{9} \frac{\rho_{p}}{\rho_{f}} \frac{r^{2}}{v}, \\
f & =\left\{\begin{array}{l}
1, \\
1+0.15 R e_{p}^{0.687},
\end{array}\right. \\
R e_{p} & =r|\mathbf{s}-\mathbf{S}| / v,
\end{aligned}
$$

where $\rho$ is density (subscript indicates particle or fluid), $r$ is particle radius, and $\mathbf{s}$ and $\mathbf{S}$ are fluid and particle velocities, respectively. $f=1$ corresponds to Stokes drag and the lower expression for $f$ incorporates an empirical correction used in Sect. 4.4 [7, 18].

As explained in Sect. B.1, the model quantities corresponding to the components of $\mathbf{s}$ in (17) depend on whether the particle is being advanced during particle-eddy interaction or during the $T$ process, and if the former, on whether it is the first or second drag-law integration during the interaction. This explains the use of a notation in (17)-(21) that does not indicate how $\mathbf{S}$ and $\mathbf{S}$ are defined in terms of model variables. When the corrected $f$ is used, (21) is evaluated based on $\mathbf{S}=(u, v, w)$ and $\mathbf{S}=(U, V, W)$ in all instances. Thus, the ODT $v$ velocity affects particle advancement when (21) is used, but not otherwise (see Sect. B.1).

During flow evolution, triplet maps are applied to velocity profiles discretized on the mesh, so the maps are implemented numerically as permutations of the discrete values, thus automatically satisfying the conservation properties of the model, which is defined mathematically as spatially continuous. Particle kinematics requires finer spatial resolution near walls than flow evolution requires. Therefore it is advantageous to exploit the Lagrangian representation of particles by using the continuum definition of the triplet map, (11), to evaluate the fluid displacements that determine fluid velocities seen by particles during particle evolution.

\section{B.4 Perspective}

It has been noted that the particle-eddy-interaction sub-model formulated here is closely related to previous formulations of this type $[6,7]$. Interaction sub-models within steady-state turbulence models typically involve a supplementary stochastic representation of eddies that interact with the particles, but in ODT, these eddies are intrinsic to the turbulence model itself. Particle response to the eddies in such models is conceptually the same as in ODT (drag-law integration, etc.).

ODT has three key distinguishing features relative to these models. First, ODT resolves all relevant flow scales and the corresponding range of eddy sizes within the turbulent cascade, but only the large eddies are treated in steady-state models (which are multidimensional, so the computational cost of fine-scale resolution is an obstacle). Therefore 
ODT is applicable over the full range of particle inertia (provided that the relevant drag-law phenomenology is incorporated); at low inertia, the small eddies are the main contributors to particle slip. This breadth of applicability enables parameter setting at low inertia before applying the model to high inertia (Sect. 4). Second, the eddy sequence in ODT provides a detailed representation of flow unsteadiness $[11,12,14]$ that contributes to the fidelity of simulated particle advancement. Third, because the eddies are the mechanism of advection in the model, scalar fluid properties are subject to the same fluid flow as the particles, thereby enforcing fine-grained consistency of the evolution of particles and their surrounding thermochemical environments. This suggests that the present formulation will be particularly advantageous for applications strongly influenced by complicated microphysical couplings.

In fact, the present formulation was developed with such applications in mind, and a preliminary version was applied to droplet burnout in a turbulent combustor. The model reproduced previously unexplained parameter dependences of droplet trajectory statistics [19]. For the channel-flow configuration considered here, model results for mean and fluctuation profiles of particle velocity components have been compared to DNS results [20, 22].

\section{References}

1. Chen, M., McLaughlin, J.B.: A new correlation for the aerosol deposition rate in vertical ducts. J. Colloid Interface Sci. 169, 437-455 (1995)

2. DeGraaff, D.B., Eaton, J.K.: Reynolds-number scaling of the flat-plate turbulent boundary layer. J. Fluid Mech. 422, 319-346 (2000)

3. Farmer, R.A.: Liquid droplet trajectories in two phase flow. Ph.D. Dissertation, MIT (1969)

4. Gioia, G., Chakraborty, P.: Turbulent friction in rough pipes and the energy spectrum of the phenomenological theory. Phys. Rev. Lett. 96, 44502.1-4 (2006)

5. Graham, D.I.: Development of particle dispersion characteristics from arbitrary initial conditions in isotropic turbulence. J. Fluid Mech. 501, 149-168 (2004)

6. Graham, D.I., James, P.W.: Turbulent dispersion of particles using eddy interaction models. Int. J. Multiph. Flow 22, 157-175 (1996)

7. Guha, A.: Transport and deposition of particles in turbulent and laminar flow. Annu. Rev. Fluid Mech. 40, 311-341 (2008)

8. Hoyas, S., Jimenez, J.: Scaling of the velocity fluctuations in turbulent channels up to $R e_{\tau}=2003$. Phys. Fluids 18, 11702.1-4 (2006)

9. Kalda, J., Morozenko, A.: Turbulent mixing: the roots of intermittency. New J. Phys. 10, 093003.1-11 (2008)

10. Kerstein, A.R.: Linear-eddy modeling of turbulent transport. Part VI: Microstructure of diffusive scalar mixing fields. J. Fluid Mech. 231, 361-394 (1991)

11. Kerstein, A.R.: One-dimensional turbulence: Model formulation and application to homogeneous turbulence, shear flows, and buoyant stratified flows. J. Fluid Mech. 392, 277-334 (1999)

12. Kerstein, A.R.: ODT: Stochastic simulation of multi-scale dynamics. Lect. Notes Phys. 756, 291-333 (2009)

13. Kerstein, A.R., Krueger, S.K.: Clustering of randomly advected low-inertia particles: A solvable model. Phys. Rev. E 73, 25302.1-4 (2006)

14. Kerstein, A.R., Ashurst, W.T., Nilsen, V., Wunsch, S.E.: One-dimensional turbulence: Vector formulation and application to free shear flows. J. Fluid Mech. 447, 85-109 (2001)

15. Liu, B.Y., Agarwal, J.K.: Experimental observation of aerosol deposition in turbulent flow. J. Aerosol Sci. 5, 145-148 (1974)

16. McCoy, D.D., Hanratty, T.J.: Rate of deposition of droplets in annular two-phase flow. Int. J. Multiph. Flow 3, 319-331 (1977)

17. McLaughlin, J.B.: Aerosol particle deposition in numerically simulated channel flow. Phys. Fluids A 1, 1211-1224 (1989)

18. Rowe, P.N.: The drag coefficient of a sphere. Trans. Inst. Chem. Eng. 39, 175-181 (1961)

19. Schmidt, J.R.: Trajectories of evaporating droplets in a turbulent combustor using the One Dimensional Turbulence model. M.S. Thesis, Univ. of Arizona (2000)

20. Schmidt, J.R.: Stochastic models for the prediction of individual particle trajectories in One Dimensional Turbulence flows. Ph.D. Dissertation, Univ. of Arizona (2004) 
21. Schmidt, R.C., Kerstein, A.R., Wunsch, S.E., Nilsen, V.: Near-wall LES closure based on OneDimensional Turbulence modeling. J. Comput. Phys. 186, 317-355 (2003)

22. Schmidt, J.R., Wendt, J.O.L., Kerstein, A.R.: A novel method for the prediction of particle-laden turbulent channel flow using the One Dimensional Turbulence model. In: Proc. 4th Int. Conf. on Heat Transf., Fluid Mech., and Thermo., Cairo, Egypt (2005)

23. Schmidt, J.R., Wendt, J.O.L., Kerstein, A.R.: Prediction of particle laden turbulent channel flow using One Dimensional Turbulence. In: Balachandar, S., Prosperetti, A. (eds.) Computational Approaches to Multiphase Flow, pp. 433-441. Springer, Berlin (2006)

24. Shin, M., Lee, J.W.: Memory effect in the Eulerian particle deposition in a fully developed turbulent channel flow. J. Aerosol Sci. 32, 675-693 (2001)

25. Shin, M., Kim, D.S., Lee, J.W.: Deposition of inertia-dominated particles inside a turbulent boundary layer. Int. J. Multiph. Flow 29, 893-926 (2003)

26. Uijttewaal, W.S.J., Oliemans, R.V.A.: Particle dispersion and deposition in direct numerical and large eddy simulations of vertical pipe flows. Phys. Fluids 8, 2590-2604 (1996)

27. Wang, Q., Squires, K.D., Chen, M., McLaughlin, J.B.: On the role of the lift force in turbulence simulations of particle deposition. Int. J. Multiph. Flow 23, 749-763 (1996)

28. Young, J., Leeming, A.: A theory of particle deposition in turbulent pipe flow. J. Fluid Mech. 340, 129159 (1997) 